\title{
Un determinante con contenido: el caso de su/sus
} A determiner with meaning: The case of $s u / s u s$

\author{
Armando Mora-Bustos* \\ Universidad Autónoma Metropolitana-Iztapalapa, Ciudad de México, México \\ ORCID: https://orcid.org/0000-0003-0743-4744 \\ Alejandra Itzel Ortiz Villegas** \\ Instituto de Educación Media Superior, Ciudad de México, México \\ ORCID: https://orcid.org/oooo-0002-4107-4968 \\ H. Antonio García Zúñiga*** \\ Instituto Nacional de Antropología e Historia, Mérida, México \\ ORCID: https://orcid.org/o0o0-0002-5247-8124
}

DoI: http://dx.doi.org/I0.28928/ri/8620I9/atcI/moraa/ortizvillegasai/garciaa

\begin{abstract}
Resumen
En el español de la Ciudad de México es frecuente que el proclítico su/sus aparezca modificando nominales de diferente naturaleza semántica. En el presente trabajo se muestra que esta forma, estrictamente funcional, funge dentro de la frase nominal como un determinante con contenido. La finalidad es mostrar que las formas $s u / s u s$ tienen una serie de rasgos semánticos binarios (definitud/anáfora-catáfora, posesión/ relación, proximidad/empatía), los cuales se distribuyen en una escala jerárquica, de manera tal que se activan o se ponen en relieve a partir del condicionamiento contextual de la frase nominal. En esta propuesta, la posesión es entendida como una relación que se establece entre una entidad que aparece en el dominio del poseedor $y$ una entidad que puede ser poseída o transferida. Los rasgos de $s u / s u s$ se interpretan en frases nominales simples y complejas a partir de la revisión del Proyecto para el Estudio Sociolingüístico del Español de España y de América (preseea) y de otro corpus integrado por registros orales de personas con escolaridad media y superior. Se muestra que, en frases del tipo su celular de Ramón, la forma su codifica definitud/ catáfora, mientras que la preposición de expresa la relación de posesión. Lo anterior deja al descubierto hallazgos relevantes para brindar una explicación detallada ante la polisemia de los proclíticos posesivos de tercera persona.

Palabras clave: frase nominal, definitud, deixis, posesión, relación, proximidad, empatía
\end{abstract}

\section{Abstract}

The proclitic su/sus modifies nouns of different semantic properties. In this paper it is shown that this proclitic is a determiner with meaning. It encodes a set of binary features (definiteness / anaphora-cataphora, possession / relation, proximity/empathy); those features are distributed on a hierarchical scale. They are activated on contextual discourse. In this proposal, possession is understood as a relationship that is established between an entity that appears in the possessor's domain and an entity that can be owned or transferred. The su/sus features are described in simple and complex noun phrases; in a noun phrases like su celular de Ramon, su encodes definiteness / cataphora, the preposition de expresses the possession. The corpus used in this study is Proyecto para el Estudio Sociolinguístico del Español de España y de América (PRESEEA). It was used a second corpus, it is composed of high school and university students' conversations. The results presented show relevant facts to provide a detailed explanation of the polysemy of third-person possessive proclitics.

Keywords: Nominal phrase, definiteness, deixis, possession, relation, proximity, empathy

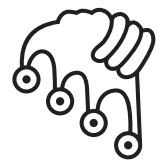

IZTAPALAPA

Agua sobre lajas

$$
\begin{aligned}
& \text { *amora@xanum.uam.mx } \\
& \text { **aov_26@yahoo.com } \\
& \text { ***agartzea@gmail.com }
\end{aligned}
$$




\section{Introducción}

Las frases nominales (FN) con los proclíticos su/sus cuentan con varias particularidades. En primer lugar, aparecen en diferentes contextos gramaticales, ya sean estos con un sentido asertivo, interrogativo, dubitativo o negado, tales como los siguientes: con complemento de régimen prepositivo (Ia), como objeto directo (Ib), en una escindida (IC), como adjunto (Id), como sujeto (Ie).

I. a. Habló firmemente con su bijo

b. En octubre comenzamos a ponerle sus hojas a la piñata y todo lo que lleva

c. Lo que ella pierde son sus ganancias

d. El muchacho me llevó hasta su casa

e. Su tatuaje se ve completamente diferente

En segundo lugar, el núcleo de la frase puede ser modificado mediante diferentes estrategias: cláusulas de relativo (2a), frases nominales con múltiples modificadores (2b) y (2c), frases nominales coordinadas $(2 \mathrm{~d})$.

2. a. Sus clientes que tiene él vienen todos los viernes

b. De sus mismos ingresos pagaba trabajadores y todo

c. ¿Y sus otros hermanos también hacían cosas así?

d. Es obviamente un sueño de una familia: su casa, su terreno, sus hijos y sus nietos; nada más

De igual manera, todas las entidades que aparecen como núcleo son susceptibles de aparecer con la marca su/sus; esto es, entidades espaciales (consultorio, casa, taller); entidades concretas (granito, carro, mantequillas); periodos de tiempo (temporada, vida, niñez); dimensiones (forma); entidades animadas (compañero, mayordomo); cualidades (máximo, estilo); parentesco (abuelo, esposa, mamá); partes del cuerpo (mano, ojo, corazón); conjunto, grupo o colectivos (familia, grupo folclórico); 
entidades abstractas (necesidades, inscripción, obligaciones); actividades (trabajo, decoración); sentido figurado o frases hechas (su quien sabe, por su bien de ella, su pinche madre), etcétera.

En tercer lugar, las formas su/sus aparecen en frases nominales en donde la relación que se establece entre poseedor y poseído está mediada por la preposición de (posesivos duplicados o frases nominales sobrespecificadas, véase Company 199I; Company y Huerta 20I7, respectivamente), como en (3).

3. a. Me gustaban mucho sus clases de ella

b. Cuando llegué de allá de mi tierra, de doce años, llevaba su regalo de ella

c. Ella, por decir, cobra todo su dinero de ella

d. Su estilo de él no me gusta mucho

En este sentido, las formas $s u / s u s$ aparecen igualmente en frases nominales como en (4); en donde su/sus se encuentran modificando tanto a la parte como a su respectivo todo, o bien, al contenido como a su contenedor.

4. a. Les quitaron sus cobijas de su cama

b. Se cayeron sus tornillos de su carro

c. Sacó sus lápices de su caja

Por último, su/sus aparece en formas lexicalizadas, como las de (5).

5. a. Él era su gallo, pues

b. Ay sí, dormía a sus horas

c. Tienen su maña todo eso

d. En su pobre casa nos levantamos temprano

El propósito de este trabajo es mostrar que las formas $s u / s u s$ tienen una serie de rasgos semánticos que se configuran en torno a la frase nominal. A partir de lo anterior, se establece la función sintáctica de $s u / s u s$, esto tanto en las frases nominales simples como en las complejas. Las interrogantes que orientan la investigación son las siguientes: ¿qué tipo de relación semántica se establece entre la forma su/sus y la entidad que denota el nominal? y ¿cuáles son los rasgos sintácticos y semánticos del determinante su/sus? Los ejemplos que se presentan han sido tomados de dos fuentes: la primera corresponde a la parte referente a la Ciudad de México del Proyecto para el Estudio Sociolingüístico del Español de España y de América 
(PRESEEA), en tanto que la segunda se trata de material recolectado en el último trimestre del 2017 por los autores de este análisis en escuelas públicas de los niveles medio y superior. Para efectos de señalar la procedencia de los datos, se decidió marcar únicamente el segundo de los corpora mediante las letras OMG. La selección de los ejemplos estuvo determinada a partir de la aparición de las formas su/sus dentro de una frase nominal simple o compleja. Por ahora, no es parte de nuestro interés realizar un trabajo de índole cuantitativa, sino de identificar y mostrar el comportamiento funcional de estas formas.

El trabajo está organizado de la siguiente manera: en el siguiente apartado se delinean los objetivos que orientan la presente investigación. Posteriormente se dan a conocer los antecedentes, es decir, los estudios más importantes que se han hecho sobre las formas su/sus; asimismo, en esa sección se justifica la propuesta y se especifican sus alcances; en la tercera parte se sientan las bases para definir el proclítico su/sus como un determinante; en el apartado que sigue se describe cada uno de los rasgos que se codifican en el determinante su/sus: relación, posesión, definitud, anáfora/catáfora, proximidad y empatía; por último, se explica la correlación de los rasgos y se plantean las conclusiones del análisis.

\section{Objetivos}

En este trabajo se plantea que los proclíticos su/sus son determinantes con contenido, es decir, que expresan una serie de rasgos: definitud, deixis (anáfora, catáfora), posesión, relación, proximidad y empatía. Estos rasgos se actualizan en cada construcción conforme a una jerarquía que define los valores dependiendo de las relaciones que se establecen entre las formas su/sus y la entidad modificada. De igual manera, dichos rasgos coocurren (coaparecen) y covarían de tal forma que, en ciertos contextos, el peso semántico se modifica dependiendo del tipo de construcción. Tomando como punto de referencia el contenido gramatical de estos rasgos, se los ha distribuido en la jerarquía de (6):

6. Definitud $>$ anáfora/catáfora $>$ posesión $>$ relación $>$ proximidad $>$ empatía

Así pues, a partir de este conjunto de rasgos, en este trabajo se señalará que en las construcciones de (7) y (8), el determinante su/sus debe ser interpretado de manera particular; esto es, los rasgos que estos proclíticos codifican varían con respecto a la construcción en la que aparecen. 
7. a. Tenía problemas con su diabetes, con su vista

b. Ya dejaron su tierra

c. Le arreglaron sus uñas, le pusieron su esmalte, su brillo

d. Por este rumbo pasaba a su casa

8. a. Esta es su casa de ellos (omG)

b. Se le cayó su tapa de su botella (омG)

c. Le compró su carro a su bijo

En la variedad del español de la Ciudad de México que aquí se describe las construcciones ejemplificadas en (7) y (8) son de uso común. El supuesto teórico que subyace en las frases que aparecen con las formas su/sus es que no siempre son las mismas y que no necesariamente codifican posesión. Por ejemplo, los rasgos presentados en el esquema de (6), definitud y anáfora/catáfora, se exhiben de manera constante, se traslapan; mientras que el resto covaría con respecto a la entidad del nominal con el que coaparecen, se reordenan y en algunos contextos se cancelan. De esta manera, la jerarquía de los rasgos del determinante su/sus en las frases nominales de (7) se estructuran como en (9); esto es, su diabetes (9a), su vista (9b), su tierra (9c) y $s u$ esmalte, su brillo (9d) y su casa (9e).

9. a. Definitud/anáfora $>$ proximidad [antipática]/( $>$ relación/posesión)

b. Definitud/anáfora $>$ relación/(posesión $>$ proximidad/empatía)

c. Anáfora/definitud $>$ empatía/(proximidad $>$ relación/posesión)

d. Anáfora/definitud $>$ relación/(posesión $>$ proximidad/empatía)

e. Definitud/anáfora $>$ posesión/(proximidad > empatía/relación)

En las frases complejas de (8), las formas su/sus codifican, igualmente, los rasgos jerarquizados tal y como se señala en (Io): su casa de ellos (Ioa), su tapa (Iob) de su botella (Ioc), su carro (Iod) y a su bijo (Ioe).

IO. a. Definitud/catáfora $>$ (posesión/relación $>$ proximidad/empatía)

b. Definitud/catáfora $>$ (posesión/relación $>$ proximidad/empatía)

c. Anáfora/definitud $>$ posesión/(relación $>$ proximidad/empatía)

d. Definitud/catáfora $>$ posesión/(relación $>$ proximidad/empatía)

e. Definitud/anáfora $>$ relación/(posesión $>$ proximidad/empatía) 
Cabe destacar que las frases de (8a) y (8b) están formadas por dos entidades; así que la relación que se establece entre ellas es de diferente naturaleza semántica. En (8a), la relación que se establece entre el poseedor y lo poseído es de posesión, pero esta se encuentra codificada por la preposición de; mientras que la relación que se genera entre las entidades de ( $8 b)$ es de parte-todo, la cual, de igual forma, está codificada por la misma preposición de. Las dos entidades de estas frases nominales forman parte del mismo constituyente. En tanto que las frases con la forma su, como la de (8c), forman parte de un constituyente diferente. Nótese que, aunque no es relevante para el análisis semántico que aquí se hace, la diferencia se centra, en términos sintácticos, en que en un caso se tiene un objeto directo, en tanto que, en el otro, lo que se tiene es un objeto indirecto.

La idea de que los determinantes codifican rasgos ha sido retomada del modelo de la gramática generativa, explícitamente de Ghomeshi, Paul y Wiltschko (2009) quienes describen este fenómeno en tres lenguas de familias lingüísticas diferentes: blackfoot (algonquina), alemán (germánica) y halkomelem (salish de la costa central). Sin embargo, el desarrollo descriptivo de este tema está fundamentado en la gramática tipológica funcional de Givón (200I), Hopper y Thompson (1980), Comrie (1981) y Shopen (1985), entre otros.

En suma, el aporte de este trabajo radica en que las formas su/sus, que tradicionalmente han sido clasificadas exclusivamente como determinantes posesivos, son determinantes con contenido, o sea, codifican un elevado grado de definitud, valores semánticos, tipos de relaciones y una especificación de sentido en la frase nominal; la diversificación semántica se da independientemente de si la construcción está duplicada.

\section{Antecedentes de los estudios de las formas su/sus}

La bibliografía que trata de manera específica las frases nominales que son materia de análisis en este trabajo, en realidad no es tan extensa. Incluso, Company (1994) reconoce que esta estructura, a la que ella en un primer momento llama "construcción nominal de apariencia posesiva", ha pasado prácticamente inadvertida en los estudios sobre posesión y en los de sintaxis española. En este apartado se sintetizan las ideas que se han postulado en torno al tema, con la intención de facilitar su comprensión y, a la vez, cimentar la propuesta de la presente investigación; a saber, que el proclítico $\mathrm{su} / \mathrm{sus}$ es un determinante con contenido semántico, el cual se reformula gracias a la interacción de un conjunto de rasgos en un contexto determinado. 
Las obras consultadas (Company, 1994; 1995; 200I; Picallo y Rigau, 1999; Huerta, 2003; Company y Huerta, 2017; Eguren 2018) ofrecen un panorama del fenómeno, el cual va desde su caracterización formal hasta las implicaciones que tiene en la gramática de la lengua, pasando por la justificación de su estudio, así como las perspectivas desde las cuales se ha analizado. Así pues, las frases en cuestión (ahí es su bodeguita de ella) se distinguen por marcar en dos lugares del mismo sintagma la referencia al poseedor. Algunos autores han argumentado que esto es necesario puesto que se trata de un mecanismo que elimina la ambigüedad generada por la polisemia referencial del proclítico $s u / s u s$, ya que, como se sabe, este elemento no indica persona, número, ni género del poseedor. La misma Company (1994) reconoce que esta hipótesis es correcta; sin embargo, inmediatamente advierte un problema: en efecto, esta explicación es válida para explicar el origen de la construcción, pero no permite entender los motivos por los cuales solo cierto tipo de poseedores requieren la duplicación. Para Picallo y Rigau (1999) el doblado del posesivo está condicionado por restricciones semánticas, esto es, el poseedor debe ser humano o susceptible de ser tratado como tal; sin embargo, esta explicación tampoco es satisfactoria de acuerdo con el tipo de construcciones encontradas en el corpus. Por su parte, Eguren (2018) explica que la construcción duplicada se reinterpretó como una construcción que establece una relación intrínseca entre el poseedor y el poseído desde el punto de vista del hablante, perspectiva relevante para entender el rasgo de empatía que se explicará más adelante.

Company (1994) explica que "la construcción posesiva con doble referencia al poseedor" resulta de interés por tres razones principales (Company, 1994ः I13). En primer lugar, debido a que el español pertenece a un grupo de lenguas en las que también se puede indicar una relación posesiva mediante una doble marca a la referencia del poseedor en la misma frase. En segundo lugar, guarda semejanzas formales con otras estrategias duplicativas de la gramática del español, tales como la valencia (aquesta lid yo la he de arrancar), la locación (aqui en este lugar) y la negación (ninguno non osava). En tercer lugar, lo que resulta de mayor relevancia para la propuesta del presente estudio, tiene una oposición contrastiva (distribución complementaria) con la construcción nominal la casa de Juan.

Algo que resulta relevante considerar es que no todas las variedades americanas del español utilizan actualmente esta estructura. Es más, en algunos países incluso son desconocidas, y en otros, estigmatizadas. En este sentido, en palabras de Company (1995: 188), posiblemente "los hablantes indígenas que aprendían español empezaron a usarla con más frecuencia porque las FN duplicadas mostraban afinidades estructurales con sintagmas y mecanismos posesivos obligatorios en sus 
respectivas lenguas, de manera que la asociación a habla de indígenas retrajo cada vez más su uso en ciertos estratos sociales". Ahora bien, Company y Huerta (2017: I84) agrupan en cinco los puntos de vista a partir de los cuales se han tratado de entender los sintagmas del tipo $s u \mathrm{~N}$ de $\mathrm{N}$ de la siguiente manera:

a. Como construcciones desambiguadoras (lo que ya se indicó más arriba).

b. Como construcciones redundantes (Rodríguez, 1982; Inzunza, 2009; Risco, 2013).

c. Como construcciones arcaizantes del español americano (Pozzi-Escot, 1973).

d. Como préstamo o contacto del español americano con las lenguas amerindias (Escobar, 1992; Granda, 1997, 1999).

e. Como construcciones con una finalidad discursivo-pragmática específica.

Es importante anotar que las hipótesis (c) y (d) tienen mucha fuerza. Al respecto, Company (1995) muestra que la estructura su $N$ de $N$ en los países con bilingüismo o fuerte contacto del español con lenguas amerindias es cuatro veces más frecuente que en aquellos países donde la presencia indígena es débil o nula, aunque también advierte que la semántica y pragmática de la doble posesión en español no coincide necesariamente con la semántica de la doble posesión en las lenguas de adstrato estudiadas. Por lo anterior, la autora sostiene que la hipótesis de contacto no es concluyente o, por lo menos, es muy difícil de comprobar. En su opinión, parece más conveniente hablar de una posible activación de la sintaxis americana por convergencia comunicativa con las lenguas amerindias, y no como un efecto del contacto.

Como se puede apreciar, el tema comporta una gran complejidad. Lo primero que se debe notar es que, en efecto, se trata de una estructura peculiar ya que es de un comportamiento poco posesivo, la cual, además, según Company (1994), tiene numerosas restricciones, de ahí su escaso empleo. En la obra referida se señala que lo más atípico es el hecho de que la construcción muestre una preferencia por poseídos humanos. En este sentido, habría una nivelación entre poseído y poseedor, en la cual la prominencia semántica de este último disminuye y, por tanto, se presenta una aminoración de la asimetría que se requiere en toda relación posesiva. De esta forma, se puede inferir que el sintagma su $N$ de $N$ está especializada para un control débil (Company, 1994: 192; Huerta, 2003). En otros términos, con estas frases posesivas el hablante indica que el poseído se encuentra muy cercano al dominio del poseedor, por lo que constituye una parte importante de él. Así, los dos miembros de la relación posesiva adquieren una relación de inherencia y proximidad conceptual tal, que en ocasiones el poseído es la cualidad esencial del poseedor. 
Lo anterior equivale a decir que el empleo de una duplicación posesiva se vincula estrechamente con el modo de conceptualizar un evento (Company, 1994: 125). Algo relevante es que, si tanto el poseedor como el poseído son entidades abstractas, el grado de control del poseedor sobre el poseído es nulo. En consecuencia, el poseído perfila una parte del poseedor, es decir, pone de relieve un rasgo atribuible a una entidad. Otras características importantes de la construcción que aquí se está describiendo son las siguientes (Company, 1994: 120, 124): la posesión duplicada es renuente a la cuantificación de sus miembros, mientras que la no duplicada parece ser indiferente a rasgos de cuantificación y el rasgo [+ determinación] es definitorio (el poseedor no es altamente determinado, la construcción no es posesiva sino especificativa). Por tanto, una consecuencia de la individualización y la determinación de los miembros de la construcción posesiva duplicada es que esta es siempre referencial y no admite ni poseedores ni poseídos genéricos. Según esta autora, nunca se documentan en el español mexicano actual FN del tipo *sus problemas de la gente o *sus hijos de las mujeres.

En términos diacrónicos, Huerta (2003: 183-184) señala que la posesión nominal ha atravesado por un complejo proceso de gramaticalización en el que el pronombre posesivo de tercera persona $s u(s)$ "ha desgastado sus significados referenciales más concretos y ha creado significados más abstractos, redefiniendo su capacidad de relacionar entidades". El primer cambio producido con $s u(s)$ fue la generalización de la posesión con FN poseídas periféricas abstractas, en tanto que el segundo fue un progresivo debilitamiento del valor de control y relacionalidad de las entidades codificadas por el pronombre posesivo (Huerta, 2003: 186). En el camino, como indicó Company (1994), se produjo una proximidad léxica entre poseedor y poseído y, a la vez, aumentó la frecuencia de uso de entidades poseedoras no humanas relacionadas con poseídos abstractos (Huerta, 2003: 194). En conclusión, la ruta del cambio se puede entender como una evolución de lo más relacional hacia lo menos relacional y de lo concreto hacia lo abstracto.

A manera de recapitulación. Las obras que se han resumido en esta sección centran su análisis en las frases del tipo su $N$ de $N$, en el tipo de referencia de los dos nominales (poseídos humanos y abstractos, por un lado, y poseedores no humanos, por el otro) y en la relación que se establece entre estos. En contrapartida, el planteamiento que aquí se hace se fundamenta en la forma su/sus; o sea, con este análisis se puede dar cuenta de las FN simples y compuestas que contengan el proclítico en cuestión debido a su función y su contenido. A lo largo de la exposición de los datos y argumentos de la presente propuesta podrá atestiguarse que la construcción con $s u / s u s$, contrario a lo que se dice en la bibliografía sobre el tema, se destaca porque: 
a. Su uso no es escaso.

b. No tiene ningún tipo de restricción; es decir, puede aparecer con cualquier tipo de entidad, sin importar su naturaleza semántica, y no solo con poseídos humanos.

c. No es renuente a la cuantificación de sus miembros.

\section{El proclítico $s u / s u s$ como determinante}

Las frases nominales de (II) están formadas por el proclítico su/sus y el núcleo nominal. Estos clíticos, de manera similar a lo que ocurre con los artículos y los demostrativos (I2), se denominan determinantes. Nótese que la presencia de un determinante está condicionada por muchos factores gramaticales: semántica del verbo o del nominal, y el estatus de la FN como relación gramatical (13). La agramaticalidad de estos últimos ejemplos está determinada por la ausencia de un determinante.

II. a. Ya tiene su consultorio

b. ¡Ah, o sea! ¿Dejó su taller?

c. El año pasado estaba haciendo su internado

d. Él es de Nayarit, ahorita ya está en su tierra

I2. a. Ya tiene el/este/ese/aquel consultorio

b. ¡Ah, o sea! ¿Dejó el/este/ese/aquel taller?

c. El año pasado estaba haciendo el/* este/ ${ }^{*}$ ese $/{ }^{*}$ aquel internado

d. Él es de Nayarit, ahorita ya está en *la/??esta/* esa/*aquella tierra

13. a. Ya tiene consultorio

*b. ¡Ah o sea! ¿Dejó taller?

${ }^{*}$ c. El año pasado estaba haciendo internado

*d. Él es de Nayarit, ahorita ya está en tierra

Los clíticos su/sus, al pertenecer al grupo de los determinantes, hacen parte de las categorías funcionales, en el sentido de que forman inventarios cerrados y sus funciones son estrictamente gramaticales. Aquí se entiende la etiqueta de determinante como una posición en la estructura gramatical que se llena con varios elementos (categorías), los cuales especifican los rasgos sobre cómo se debe identificar el referente de un sustantivo particular (o, en algunos casos, la clase de relación que tiene 
este con algún tipo de entidad). Los determinantes incluyen artículos, demostrativos, pronombres posesivos, algunas formas QU, pronombres, etc. (Payne, 2006). El determinante, como la mayoría de las categorías funcionales, es una unidad prosódicamente no acentuada y posee un contenido funcional, en el sentido de que no denota seres o entidades.

Si bien en la literatura hay un acuerdo generalizado sobre la función modificadora del determinante sobre el núcleo nominal, poco se señala con respecto a este carácter modificador. Van Valin y La Polla (1997) se inclinan por mencionar que el determinante modifica el sustantivo en cuanto a sus rasgos referenciales. Por su parte, Dryer (2007) menciona que en el Batad Ifugao (lengua de las Filipinas), las frases nominales ocurren con determinantes que codifican una variedad de propiedades gramaticales que interactúan con el sistema de foco. En la descripción de Dryer (2007) no se hace alusión al tipo de propiedades gramaticales que se codifican en el determinante. Bosque y Gutiérrez-Rexach (2008:6I4) señalan que los determinantes, como indica su etiqueta categorial, "determinan" o "especifican" la referencia del nombre sobre el que inciden, es decir, precisan su cantidad, su referencia u otras propiedades relacionadas con estas, como son las de posesión, anáfora/catáfora, etc. En el trabajo que aquí se presenta, como ya fue mencionado anteriormente, el determinante se considera una categoría funcional, la cual, efectivamente, no detona un contenido léxico, pero sí codifica un conjunto de rasgos o propiedades gramaticales, como en (I4).

14. a. Definitud
b. Anáfora/catáfora
c. Posesión
d. Relación
e. Proximidad
f. Empatía

La susceptibilidad de los determinantes, en este caso en particular de su/sus, para codificar un conjunto de rasgos permite, en parte, dar cuenta del porqué su/sus aparece, de manera general, modificando nominales que expresan sentidos de diferente naturaleza semántica; esto es, los sustantivos que denotan partes del cuerpo ( $15 \mathrm{a})$, parentesco ( $15 \mathrm{~b}$ ), que, como se mostrará más abajo, no codifican en estricto sentido posesión, entidades susceptibles de ser poseídas (I6a), entidades que denotan un sentido de proximidad más que de posesión (I6b), entidades sobre las que se 
expresan sentimientos de empatía (I6c), y entidades que, por el contrario, expresan un sentido de antipatía (I6d).

15. a. A Pedro le operaron de emergencia su corazón

b. Llamó a su bijo para que venga a recogerlo.

I6. a. Antonio Villa estaba haciendo su tesis de maestría (omg)

b. Llamaba ella y pues sí fuimos hasta su funeral también

c. Hoy juega su equipo de fútbol (omg)

d. Sus dolores de cabeza lo tienen en la cama (omg)

Todos estos rasgos o valores que están codificados en el determinante su/sus se actualizan en el discurso; es decir, los interlocutores van a identificar dentro del marco de la interacción comunicativa de qué tipo de referente, expresado en la frase nominal, se trata.

Junto a los rasgos ilustrados en los ejemplos (I5) y (I6), el determinante su/sus codifica la definitud (I7a) y la anáfora (I7b). Los nominales modificados por este determinante obligatoriamente son definidos (Givón, 200I) y expresan un tipo específico de referencia deíctica anafórica. En ( $17 b)$, el sujeto gramatical, él, y el determinante sus de la frase nominal objeto directo hacen referencia cruzada.

17. a. Una ocasión que le dije algo de que si podía quitar su carro

b. Él hizo sus ${ }_{i}$ cálculos estructurales

Los rasgos codificados en el determinante su/sus no tienen la misma naturaleza gramatical. La definitud y la anáfora/catáfora son intrínsecos, obligatorios; aparecen codificados por default. Estos coaparecen y coocurren libremente; mientras que el sentido de los rasgos de proximidad, posesión, relación y empatía están correlacionados directamente con la expresión discursiva. Esto hace que no coaparezcan libremente; por ejemplo, al activarse el rasgo de posesión, la proximidad, la relación y la empatía se ubican en un segundo plano o se cancelan; igualmente, al activarse el rasgo de empatía, el conjunto de rasgos restante aparece degradado o bloqueado; este hecho implica que los rasgos aparezcan distribuidos en la jerarquía de (6), nuevamente ilustrada en (18).

I8. Definitud $>$ anáfora/catáfora $>$ posesión $>$ relación $>$ proximidad $>$ empatía 
Los rasgos semánticos del determinante $s u / s u s$ que están distribuidos en la escala de (I8) no tienen el mismo estatus; la definitud y la anáfora/catáfora van a aparecer en la parte más alta de la escala, mientras que el resto de los rasgos tiene la posibilidad de moverse de derecha a izquierda y viceversa, así como de bloquearse. La prominencia dentro de la jerarquía no es solo conceptual sino configuracional; esto implica que la activación de los rasgos del determinante está asociada a la naturaleza semántica del núcleo y al contexto situacional en el que una expresión es emitida.

En las frases nominales de (19a), sus tamalitos y ( $19 \mathrm{~b}$ ) sus chiles y su mayonesa, la forma $s u / s u s$ no expresa un sentido estricto de posesión, sino expresa una relación de conjunto, es decir, sus tamalitos están relacionados con atole, mientras que sus chiles y su mayonesa se relacionan con sándwich. En este tipo de construcción, la referencia anafórica se degrada; no obstante, dentro del discurso se recupera la relación que existe entre los elementos del conjunto, así como entre la entidad contenedora y sus respectivos contenidos. La degradación de la referencia no influye en el hecho de que esta forma codifique en primer grado la definitud de la entidad a la que está modificando. Esta propiedad del determinante se observa con mayor claridad si se contrastan los ejemplos de (19) frente a los de (20).

19. a. Les dan el primer día atole con sus tamalitos

b. Le pusieron al sándwich aguacate, sus chiles, su mayonesa

Las frases nominales de (20) están integradas por el determinante su/sus y dos entidades relacionadas con la preposición de. Si bien en este tipo de frase nominal la relación que está mediada por la preposición de no presenta la misma naturaleza semántica y sintáctica, el determinante su/sus no codifica posesión, sino que simplemente expresa la definitud y la anáfora/catáfora; en (20) el sentido de posesión o relación está expresado por la preposición de.

20. a. Sí, ese es su único vicio de él (omg)

b. Pues su hermano de mi papá ha corrido tres maratones

c. Me iba contando todos sus problemas de su casa

d. Me gusta mucho el tamal de mole por ejemplo con sus rajitas de pollo 


\section{Rasgos que se codifican en el determinante}

Recuérdese que uno de los aspectos centrales de la presente propuesta es definir el proclítico su/sus como un determinante que especifica la referencia del nombre, a la vez que precisa la cantidad y la misma referencia. Este determinante tiene contenido, que se expresa mediante los rasgos de definitud, anáfora/catáfora, posesión, relación, proximidad, posesión y empatía, los cuales se actualizan en cada construcción, coocurren (coaparecen) y covarían. Al respecto es importante señalar que los rasgos cuentan con otras dos características. La primera de ellas es que se ordenan siempre en pares; esto es, la definitud se complementa con la anáfora o la catáfora; la posesión, por su parte, se opone a la relación, en tanto que la proximidad se contrapone a la empatía. La segunda de estas características es que en una frase específica hay rasgos que se cancelan. Esto hace que, en ciertos contextos, el peso semántico se modifique.

De acuerdo con la escala propuesta en (I8) la actualización de rasgos de las frases nominales se activa en el momento de la enunciación, la jerarquía tiene un desarrollo de lo menos definido (2Ia) hasta llegar a la participación afectiva (2Ih). Estos rasgos semánticos (definitud (2Ib) > anáfora/catáfora (2IC) > posesión (2Id) y $(2 \mathrm{Ie})>$ relación $(2 \mathrm{If})>$ proximidad $(2 \mathrm{Ig})>$ empatía $(2 \mathrm{Ih})$ ) tienen una correlación sintáctica, un artículo indefinido, uno definido, una marca genitiva y una forma tradicionalmente llamada posesiva que expresa, de acuerdo con la escala, más de un valor no necesariamente posesivo.

2I. a. Un pirul está en Toluca

b. El pirul está en Toluca

c. En la zona alta hay una arboleda, los eucaliptos están a la orilla del río, los cipreses cerca del río y el pirul en el centro del parque.

d. Rosa podó su pirul.

e. Le sacaron mucha resina a su pirul de Rosa.

f. La leña del pirul es muy olorosa.

g. Al biólogo le robaron la muestra de sus pirules.

h. A los habitantes de la colonia les van a cortar sus pirules para ampliar la calle.

\section{Definitud y anáfora/catáfora}

En la lengua española existe un gran repertorio de formas dentro de la frase nominal que expresan definitud del referente expresado en el núcleo nominal; en los 
ejemplos de (22), excepto (22a), los determinantes el, este, dos, algunos y su indican que el referente es identificable. Aquí se asume que dentro de la gran clase de determinantes (artículos, demostrativos, numerales, etc.), las formas $s u / s u s$, como en (22f), son inherentemente definidos; por excelencia, los que codifican mayor grado de definitud o especificidad, en consecuencia, son los que generan mayor referencia del núcleo.

22. a. Ya compré café

b. Ya compré el café

c. Ya compré este café

d. Ya compré dos cafés

e. Ya compré algunos cafés

f. Ya compré su café

La definitud es entendida como el rasgo de naturaleza semántica que constituye el contenido de un artículo definido. Las propiedades de la definitud son la familiaridad y la anáfora/catáfora. Dentro de la perspectiva de la familiaridad, el referente de una frase nominal definida es conocido por los interlocutores del acto de habla. Este referente ya debe de haber sido mencionado en el discurso. En sentido amplio, la definitud de una entidad está relacionada con el ámbito anafórico del referente en cuestión y con el conocimiento general que se tenga, por parte de los interlocutores, sobre este referente. En (23a), sus hace referencia cruzada con la referencia expresada por el cuantificador bastantes y la flexión de número plural codificada en el verbo vienen. Por su parte, la agramaticalidad de (23b) consiste en la falta de correferencia del determinante indefinido unas.

23. a. Pero, ahí llega bastante coche porque bastantes vienen $_{\mathrm{i}}$ a hacer ejercicio, a correr o en sus bicicletas

b. *Pero, ahí llega bastante coche porque bastantes vienen $_{i}$ a hacer ejercicio, a correr o en unas $_{\boldsymbol{x}_{\mathrm{i}}}$ bicicletas

La definitud y la referencia endofórica (anáfora o catáfora) se traslapan en la forma su/sus. En la construcción de (24a), se podría referir que su casa hace correferencia con mi suegro, pero esta referencia se transparenta con la extensión periférica donde iban a vivir. En esta construcción queda claro que a cada hijo le dieron una casa y no una sola para todos. Entre la forma su casa y la referencia anafórica a mis clientes 
particulares de (24b) no se establece concordancia de número. Esto no implica que no se pueda identificar a lo que refiere la forma su.

24. a. Mi suegro vivía, les dio a todos sus hijos su casa donde iban a vivir

b. A mis clientes particulares, yo voy a su casa a verlos ¿no?

El determinante su/sus liga obligatoriamente las anáforas/catáforas; en el ejemplo de (25), sus especias, hace referencia a tipo de barbacoa. En este contexto la frase con sus especies aparece coordinada con una frase nominal modificada por un artículo, el chile. Las FN modificadas por su/sus son altamente definidas; el grado de definitud está asociado con el hecho de que se correlaciona con una sola unidad en el universo del discurso.

25. a. A: ¿Y cómo la hacen? ¿Nada más, así como tipo barbacoa?

b. B: Ajá, se prepara el chile bien y con todas sus especias ${ }_{\mathrm{i} / \mathrm{j}}$ y se enchila la carne $\mathrm{y}$ ya se pone en los hornos

Las frases nominales como sus especias de (25b) presenta un aparente problema de correferencia; este estaría asociado con un sentido de ambigüedad del determinante su/sus. La referencia que establece esta forma puede estar dentro del ámbito del acto de habla (referencia exofórica) o dentro del discurso (endofórica). En el primer caso sus estaría haciendo correferencia con una tercera persona, que sería la poseedora de las especies; mientas que en el segundo sus hace correferencia con el referente chile $_{\mathrm{i}}$ y barbacoa $_{\mathrm{i}^{+}}$Con respecto a este hecho se debe señalar que, en los ejemplos encontrados en el corpus, la interpretación de la correferencia exofórica no es posible.

Dentro de las características que codifica la forma su/sus están las frases nominales de (26), en donde el rasgo de posesión aparece degradado, por lo que está expresado por la preposición de. La función, en estos contextos de su/sus, es la de un delimitador o restrictor de la referencia de los nominales casa y hermano. El rasgo de definitud expresado en esta forma está asociado a la correferencia que se establece entre las dos entidades relacionadas, $s u_{\mathrm{k}} \ldots L u p e_{\mathrm{k}}$ y $s u_{\mathrm{k}} \ldots m i p a p a_{\mathrm{k}}$.

26. a. Le gusta que $s u_{\mathrm{k}}$ casa de Lupe parezca palacio

b. $S u_{\mathrm{k}}$ hermano de mi papá ${ }_{\mathrm{k}}$ vive en el gabacho

Con todo lo expuesto se puede observar que los rasgos de definitud y anáfora/ catáfora están correlacionados. Son consustanciales en un sentido restrictor de la 
referencia de la entidad expresada en el nominal nuclear. La definitud depende del rasgo de familiaridad; el referente del nominal debe estar bajo el conocimiento de los interlocutores, de tal manera que la referencia anafórica/catafórica sea fácil de reconocer o identificar.

\section{Posesión y relación}

El determinante su/sus expresa un sentido de posesión en las FN en donde las entidades que son expresadas por el nominal pueden ser poseídas o indican una posesión transferida, esto es, antes no formaban parte del dominio del poseedor [+animado], [+/-humano], pero son adquiridas, regaladas o transferidas para que formen parte de él, como en (27a) objetos del hogar; ropa (27b); otras propiedades (27c); animales domésticos y animales de trabajo (27d); alimentos (27e); productos intelectuales o manuales (27f).

27. a. Están lavando sus platos

b. Su pantalón está colgado en el alambre

c. No quería pintar su casa

d. Le prohibieron sacar a su perro

e. Se enfriaron sus tortillas

f. Su obra no fue del agrado del público

A diferencia de ello, cuando el determinante su/sus está acompañado por un núcleo que hace referencia a partes del cuerpo como en (28) o a relaciones de parentesco como en (29), el determinante $s u / s u s$ expresa una relación parte-todo, o bien, algunas relaciones sociales (véase más adelante [33]); el determinante codifica un sentido de cercanía entre las entidades y restringe la referencia del nominal.

28. a. Le entró polvo en su ojo

b. Se fracturaron sus dos tobillos

29. a. Quiere hacer recámaras para sus hijos que están fueras

b. Sus padres no pudieron viajar por el intenso frío

El estudio sobre la posesión se ha planteado, generalmente, en términos binarios (posesión alienable e inalienable); sin embargo, desde nuestra perspectiva se asume 
que existen distintas relaciones semánticas además de la posesión. Sobre este tema se resumen cuatro planteamientos: i) un sistema binario (alienable e inalienable) (Haspelmath, 2008), ii) una perspectiva de prototipos (Seiler, 1981; Heine, 1997b), iii) un sistema de tres relaciones centrales (ownership, kinship y whole-part) (Aikhenvald y Dixon, 2013) y iv) un conjunto de relaciones semánticas diversas (Arellanes, Ortiz Villegas, Mora-Bustos y García Zúniga, 20r4; Ortiz, Arellanes, Mora-Bustos y García Zúñiga, 20I4; Ortiz, 2015; 2016).

Esta última propuesta considera que las relaciones semánticas están organizadas por dominios y rasgos caracterizadores como la cohesión de las entidades, la concepción de la unión entre estas y la animacidad de las entidades involucradas. En ese sentido, destacan cuatro dominios: en el primero se establecen relaciones entre una entidad animada y otra inanimada, tal es el caso de la posesión (30) y de la meronimia, en específico cuando se involucran partes del cuerpo, como (3I).

30. a. ¿No les dio su terrenito a las hijas?

b. Cosas que les han pasado en su vida y lo relacionan con su tatuaje

c. Cuando viví con ella en su casa, me llevé el changarrillo para allá

3I. a."Ah bueno", y pone ella su cachetito

b. Ahorita el problema fuerte que tiene es que se está descalcificando su brazo

c. Su cuello con su cuellito en cuadros cafés, su vestido amarillo

En el segundo dominio se agrupan relaciones que se establecen entre entidades inanimadas como en (32). Como puede verse, las partes de partes del cuerpo (32a), las partes de objetos inanimados (32b); las porciones (32c) o el tipo de materia (32d) dan cuenta de este dominio.

32. a. El niño se rompió su dedito del pie (OMG)

b. Van con sus hojas de palma, van así

c. En la feria le compramos su rebanada de pizza (омG)

d. Un día estaba con su vestido de lino (oмG)

En el tercer dominio se clasifican las relaciones que involucran entidades humanas, como el parentesco o las relaciones sociales no consanguíneas (33). Por último, en el cuarto dominio se establecen relaciones entre un tema y una locación, como en (34). 
33. a. Para ella lo primero lo primero es su familia

b. O sea, ya ahorita tiene su niña

c. Ya me puso también el ejemplo de uno de sus compañeros

34. a. Pidió su queso de Chiapas (omg)

b. Le entregaron su molito de Oaxaca (omg)

c. Los señores cuando van a sus campos de cultivo, van con sus hojas y van teje y teje

d. Entonces, es lógico que quieran tener su propia babitación

Siguiendo esa perspectiva, se entiende la posesión -en términos de pertenenciaagrupada dentro del primer dominio. Para esto se retoma el sentido binario mencionado anteriormente, pero desde esta postura, se establece la diferencia entre posesión alienable, la cual se caracteriza por una entidad [+/-animada] que forma parte del dominio del poseedor, como (35a), y la posesión inalienable (tradicionalmente estudiada como partes del cuerpo, como en el brazo de la señora o parentesco, como el tío de Juan), entendida aquí - a partir de estudios de lenguas amerindias- como una entidad [-animada], la cual a su vez se caracteriza por ser inherente al poseedor, como la ropa o elementos culturalmente establecidos (35b); las frases nominales que no tengan esas características desactivan el rasgo de posesión, como en (36). En el segundo dominio, por ejemplo, se hace referencia a relaciones entre partes, contenidos o sustancias (36a), en este caso, al igual que en las relaciones presentes en el tercer dominio (36b), se desactiva el rasgo de posesión y se activa el de relación dado que se involucran dos entidades que carecen de una relación de pertenencia, es decir, se caracterizan por ser [-poseedor] [-poseído]. Lo mismo ocurre en el último dominio, en el que se relaciona un tema y una locación (36c).

35. a. Y sí le compré su acordeón

b. Traía pantalón de montar, de charro [... su sombrero de caporal, su sombrero de palma

36. a. Pues le hice su vestidito de lino (omg)

b. Me dijo que ahora sí ya podía ser su novia

c. Una recámara sí estaba grande y la otra era más chica, pero sí tenían su clóset 
Cuadro i. Esquema de Relaciones en fN Simples y Complejas

(I)

Su casa de Juan

FN

Det

$N^{\prime}$

$\mathrm{N}$

FP

Su casa

Prep

FN

[Definitud/catáfora] Poseído

[Posesión]

Juan

POSEEDOR

(2)

Su tapa de su botella

FN

Det

N'

N

FP

Prep

FN

Det N

$\mathrm{Su}$ tapa

de

[Definitud/catáfora]

[Relación]

$\mathrm{su}$

botella

[Definitud/anáfora ${ }_{i / j}$ ]

[Posesión]

TODO

PARTE

${ }^{\star}$ El referente ${ }_{i}$ se encuentra en el acto de habla (elemento endofórico).

${ }^{*}$ El referente ${ }_{\mathrm{j}}$ puede ser discursivo (elemento exofórico).

De esta manera se enfatiza que la posesión hace referencia exclusivamente a la relación que existe entre dos entidades, el poseedor (preferentemente humano y animado) y una posesión [+/ - animada] con las características de (37) (Seiler, I98I; Heine, I997b; Taylor, 1999):

37. a. La relación se establece entre un poseedor [+humano]/[+animado] y un poseído [+/-animado], es una relaciónः i) exclusiva, ii) de control, iii) permanente, iv) con proximidad espacial y asociación física espacio-temporal y v) de largo término.

b. En este dominio se identifica una relación entre los seres humanos y sus pertenencias o productos culturales e intelectuales.

c. La posesión atributiva se expresa sin ninguna mediación verbal. 
En suma, el rasgo de posesión se activa en construcciones como las referidas en (27), mientras que se desactiva en FN como las de (28), toda vez que queda manifestada la inclusión de la parte (merónimo) respecto del todo (holónimo); de igual forma, en los ejemplos de (29) se desactiva el rasgo porque la única relación presente es consanguínea o de jerarquía. La escala queda resumida en (38).

38. a. Definitud/anáfora $>$ posesión/(relación $>$ proximidad/empatía)

b. Definitud/anáfora $>$ relación/(posesión $>$ proximidad/empatía)

Las frases nominales descritas hasta ahora pueden ser simples como las de (39a) o complejas como las de (39b), lo mismo ocurre cuando el rasgo de relación se activa en frases nominales simples (39c) o complejas (39d).

39. a. Sí, mira, aquí se queda, aquí está su ropa.

b. Ahí es su bodeguita de ella

c. Se tropezó y se lastimó su tobillo

d. Él le (sic) habló a sus amigos de mi hermano

\section{Proximidad}

La proximidad es un rasgo que ha servido para analizar la posesión: refiere una relación conceptual. En términos semánticos, este rasgo alude a una cercanía espacial y temporal de algo o de alguien, aunque para los términos de este trabajo debe entenderse como la distancia existente entre dos entidades en un dominio general de posesión. La proximidad es un concepto que se define básicamente a partir de dos instancias: la existencia y el mismo dominio de posesión. La existencia puede ser física o abstracta, en tanto que el dominio puede explicarse a partir de la intención o deseo de acercamiento. La relación que se establece entre dominio y existencia es de tres clases. En la primera, véase (40a), la existencia de la entidad es parcial; o sea, su tesis es una entidad que no existe totalmente y, por tanto, todavía no forma parte del dominio del poseedor. En este sentido, se puede decir que las entidades que son modificadas por el determinante su/sus en términos de proximidad, funcionan como un sustantivo contable. La diferencia con (4ob) es que la entidad ya existe, pero no está en el dominio del poseedor, pero este tiene la intención o el deseo de adquirirla. En (40c) no hay forma en que la entidad pueda ser poseída, a pesar de que exista. En (4od) la entidad existe en un plano básicamente abstracto. 
40.a. Pues a él le gusta y ya él ansía más que nada que se termine su tesis (OMG)

b. Hoy vio pasar su BMW (омG)

c. El actor está leyendo sus guiones (oMG)

d. La empleada mostró su inteligencia y su lealtad ante la jefa (omG)

En (4I) se esquematiza la relación de proximidad que existe entre las dos entidades involucradas en la posesión.

4I. a. Existe en el mundo parcialmente y puede formar parte del dominio de posesión

b. Existe en el mundo totalmente y puede formar parte del dominio de posesión

c. Existe en el mundo totalmente y nunca formará parte del dominio de posesión

d. Valores universales positivos, que no son necesariamente inherentes al individuo

En (42) se muestra la relación existente entre los rasgos propuestos para el proclítico su/sus, que sirven para analizar las frases de (40).

42. Definitud/anáfora > proximidad/ (empatía > relación/posesión)

Como se puede notar, el esquema refleja lo que sucede en las frases en cuestión. Esto quiere decir, por un lado, que solo se activan los rasgos de definitud, anáfora y proximidad, por lo que se bloquea el resto de los rasgos. Por otro lado, las diferencias entre las frases las proporcionan las características señaladas en (4I). En este sentido, solo los esquemas de los rasgos de definitud, anáfora/catáfora, relación y posesión muestran las distinciones entre las frases que se analizan.

Existe otro grupo de relaciones en las que hay algo, una enfermedad, por ejemplo, que va en detrimento del poseedor $y$ va a depender del tipo de entidad que sea susceptible de ser poseída. En este tipo de relaciones, incluso, el poseedor puede dejar de existir o tener características de valoración universal negativa. A esta relación se le denominará proximidad antipática. No se debe confundir esta proximidad antipática con aquella en la que se resaltan valores universales positivos.

43. a. Tenía problemas con su cuello y su espalda

b. No se da cuenta todo lo que le afecta su alcoholismo (omG)

c. Llamaba ella y pues sí fuimos hasta su funeral también

d. Su holgazanería no lo va a llevar a ningún lado (oмG) 


\section{Empatía}

La empatía (cf. Moya-Albiol, et al., 2010; Eguren, 2018) consiste en la identificación mental y afectiva que se genera entre el individuo y la entidad denotada, la cual está expresada por el núcleo nominal, es decir, se identifica al referente del nominal como una entidad cercana. Se marca como posesión, pero nunca se va a integrar al dominio del poseedor.

44.a. Otra vez perdió su equipo (omG)

b. Están tocando su canción (omG)

c. Ya llegó su taxi (omg)

d. ¡Shhh, cállense! que ya va a empezar su película (OMG)

En (45) se presenta el esquema de los rasgos de la empatía.

45. Definitud/anáfora $>$ empatía/(proximidad $>$ relación/posesión)

Una particularidad de este rasgo es que es más proclive a que la entidad que puede ser poseída tenga que ser singular, solo en algunos cuantos casos será posible tener un plural:

46. a. Ya fueron a comprar sus madalenas (OMG)

b. Esta semana perdieron todos sus equipos (oMG)

c. Están afuera sus taxis (cuando el sujeto es un solo individuo) (omG)

d. Hoy no van a transmitir sus telenovelas (cuando el sujeto es un solo individuo) (омG)

Una segunda característica importante de este rasgo es que remite solamente a la entidad que puede poseer porque la relación que se establece con lo poseído es estrictamente afectiva, siempre en términos positivos. Recuérdese que las afectaciones negativas se clasifican como proximidad antipática. Por último, otra característica de este rasgo es que la entidad que puede ser poseída nunca va a formar parte del dominio de la entidad poseedora. 


\section{Correlación de rasgos}

Los rasgos de la forma su/sus son jerárquicos, binarios consustanciales y se activan o se bloquean dentro de la escala. La activación significa que los rasgos dentro de la escala van a tener una orientación hacia la izquierda. Por el contrario, en la cancelación o bloqueo se orientan hacia la derecha. El bloqueo quiere decir que no están en realce los rasgos, esto no significa que no existan, más bien se encuentran degradados. De forma particular, solamente el primer par de estos rasgos binarios (definitud/anáfora-catáfora) va a aparecer en la parte más alta de la escala, porque es una propiedad inherente al determinante. Debido a esto, nunca va a ser degradado o bloqueado. Lo que sí puede ocurrir es que cambien de plano: en algunos casos iría primero la definitud y en otros la anáfora/catáfora. En cambio, el otro conjunto de rasgos (relación/posesión y proximidad/empatía) adquiere sentido en la actualización de las emisiones dentro del discurso y, de igual manera, pueden permutar su orden dentro del par correspondiente.

47. a. Me quedé en [su babitación] de mi prima (omg)
a'. Definitud/catáfora $>$ (relación/posesión $>$ proximidad/empatía)
b. Esta mañana abrió su regalo (oMG)
b. Definitud/anáfora > posesión /(relación > proximidad/empatía)
c. Le enyesaron su brazo (omG)
c.' Definitud/anáfora > relación /(posesión > proximidad/empatía)
$\mathrm{d}$. Se tiene que dormir temprano porque mañana tiene que ir a hacerse sus análisis (omG)
d'. Definitud/anáfora > proximidad/(empatía > relación/posesión)
Nadie le aguanta su mal genio (omG)
e. Definitud/anáfora > proximidad antipática/(empatía > relación/posesión)
Finalmente llegó a su ciudad natal (oMG)
f'. Definitud/anáfora > empatía/(proximidad > relación/posesión)

Esta representación es abarcadora para la variante del español aquí estudiada en dos sentidos. Primero porque, como se ha dicho, el determinante su/sus aparece modificando a entidades de diferente naturaleza semántica; es decir, no tiene ningún tipo de restricción al respecto. En segundo lugar, esta propuesta da cuenta de todas las frases nominales simples y compuestas donde aparece el determinante su/sus. Bajo este análisis, en las construcciones del tipo su cartera de José la preposición de no puede marcar ningún tipo de relación que no sea la posesión. Esto es, no puede marcar la 
empatía, la proximidad, la relación, entendida esta como compleja, pudiendo ser de parte-todo, de jerarquía social, etc. Así, pues, su marca la definitud/anáfora, ya que el resto de los rasgos no pueden aparecer en este tipo de construcciones.

\section{Conclusiones}

En este trabajo se ha presentado una descripción de las FN que aparecen codificadas por el determinante su/sus. La importancia de esta propuesta radica en el hecho de que este proclítico codifica un conjunto de rasgos binarios (definitud/anáfora-catáfora, posesión/relación, proximidad/empatía) que se activan dependiendo de la frase nominal referida. En este sentido la propuesta que aquí se ha desarrollado se distancia de otros planteamientos, en el sentido de que un determinante o una categoría funcional tiene contenido semántico.

En esta propuesta de trabajo se asume que el pronombre posesivo no solamente codifica posesión. Esta idea subyace en el concepto mismo que se ha asumido para dar cuenta de lo que se entiende por posesión; esto en un sentido muy amplio, en donde cabe el espectro de las entidades que se relacionan, en función de si efectivamente entran en la esfera de lo que generalmente se considera posesión. Igualmente, los conceptos de anáfora/catáfora, relación, proximidad y empatía permiten restringir la funcionalidad de este determinante en las FN objeto de estudio.

La concepción del determinante con contenido semántico ha permitido describir FN de diferente naturaleza semántica. Dentro de esta variedad de frases nominales modificadas por la forma su/sus ha sido de relevante importancia, entre otras cosas, haber intentado señalar que aquí se ha planteado un análisis diferente de las frases que dentro de la literatura especializada son consideradas como doble posesión (su ropa de la niña), en donde se contempla que la preposición de expresa la posesión y la forma su simplemente codifica un alto grado de definitud.

Finalmente, esta propuesta ha sido un primer intento para dar cuenta de las frases nominales que aparecen con la forma $s u / s u s$ en función de determinante. Sin duda, lo descrito en este texto no es una propuesta acabada y concluida; por el contrario, se han presentado hechos de lengua reveladores de un fenómeno mucho más complejo de lo que hasta el momento se ha sostenido. Lo aquí presentado debe considerarse como una provocación lingüística, una invitación o la puesta en escena de los abundantes tópicos del análisis gramatical. 


\section{Referencias bibliográficas}

Aikhenvald, Alexandra y Robert Malcolm Ward Dixon (eds.)

2013 Possession and ownership. A Cross-Linguistic Typology, Oxford: Oxford University Press.

Álvarez González, Albert

2012 "Estructuras posesivas en yaqui", Universos, 9, pp. II5-I3I.

Arellanes Arellanes, Francisco, Alejandra Ortiz Villegas, Armando Mora-Bustos y H. Antonio García Zúñiga

2014 "Syntax of the non-eventive semantic relations in four otomanguean languages: amuzgo, mazahua, mazatec and zapotec", ponencia presentada en 6th International Conference Syntax of the World's Languages, del 8-io de septiembre de 20I4, Pavía, Italia.

Bosque, Ignacio y Javier Gutiérrez Rexach

2008 Fundamentos de sintaxis formal, Madrid: Akal.

Company, Concepción

I994 "Semántica y sintaxis de los posesivos duplicados en el español de los siglos xv y xvi", Romance Philology, xLIV(4), pp.III-35.

Company, Concepción

I995 "Cantidad vs. Cualidad en el contacto de las lenguas. Una incursión metodológica en los posesivos 'redundantes' del español americano”, Nueva Revista de Filología Hispánica, 43(2), pp. 305-339.

Company, Concepción

200I "Gramaticalización, debilitamiento semántico y reanálisis. El posesivo como artículo en la evolución sintáctica del español”, Revista de Filología Española, 8I(I-2), pp. 49-87.

Company, Concepción y Norohella Huerta

2017 "Frases nominales sobrespecificadas encabezadas por un posesivo átono: su casa de Juan, su casa que tiene Juan", en: Concepción Company y Norohella Huerta (eds.), La posesión en la lengua española, Madrid: Consejo Superior de Investigaciones Científicas, pp. I77-218.

Comrie, Bernard

198I Language Universals and Linguistic Typology. Syntax and Morphology, Chicago: The University of Chicago Press. 
Dryer, Matthew

2007 "Noun phrase structure", en: Timothy Shopen (ed.), Clause Structure, Language Typology and Syntactic Description, vol. 2, Cambridge: Cambridge University Press, pp. I5I-205.

Eguren, Luis

2018 "Evaluative prenominal possessives in Spanish", Borealis: An International Journal of Hispanic Linguistics, 7(I), pp. I-26, DoI: https://doi. org/I0.7557/I+7.1.4254

Escobar, Anna María

I992 "El español andino y el español bilingüe: Semejanzas y diferencias en el uso del posesivo", Lexis, xvi(2), pp. I89-222.

Ghomeshi, Jila, Ileana Paul, Martina Wiltschko

2009 Determiners, Ámsterdam: John Benjamins Publishing Company, pp. I-2, DoI: https://doi.org/IO.IO75/la.I47.ootoc

Givón, Talmy

200I Syntax, vol. I, Ámsterdam/Philadelphia: John Benjamins Publishing. Granda, Germán de

1997 "Replanteamiento de un tema controvertido. Génesis y retención del doble posesivo en el español andino", Revista de Filología Española, Lxx$\operatorname{VII}(I-2)$, Pp. I39-I47.

Granda, Germán de

1999 "Retención hispánica y transferencia quechua en dos fenómenos morfosintácticos del español andino", Lexis, 23(I), pp. I37-I5I.

Haspelmath, Martin

2008 "Alienable vs. inalienable possessive constructions", Syntactic Universals and Usage Frecuency. Handout 3, Leipzig Spring School on Linguistic Diversity.

Haspelmath, Martin, Matthew Dryer, David Gil y Bernard Comrie (eds.) 2008 The World Atlas of Language Structures Online, Leipzig: Max Planck Institute for Evolutionary Anthropology:

Heine, Bernd

I997a Cognitive Foundations of Grammar, Oxford: Oxford University Press. Heine, Bernd

I997b Possession: Cognitive Source, Forces, and Grammaticalization, Cambridge: Cambridge University Press.

Hopper, Paul y Sandra Thompson

I980 “Transitivity in Grammar and Discourse”, Language, 56(2), pp+ 255-299. 
Huerta Flores, Norohella

2003 "Gramaticalización del posesivo. Un proceso hacia la abstracción", Medievalia, 35, pp. I83-97.

López Inzunza, Ingrid

2009 Posesivos redundantes argumentales en el español de México, tesis de maestría, Universidad Nacional Autónoma de México.

Moya-Albiol, Luis, Neus Herrero y Consuelo Bernal

2010 "Bases Neuronales de la empatía", Revista de Neurología, 50(2), pp. 89-100.

Ortiz Villegas, Alejandra

2015 "La posesión atributiva en mazateco", ponencia presentada en el XIII Congreso Nacional de Lingüística, del 7-10 de octubre de 2015, Tuxtla Gutiérrez, Chiapas.

Ortiz Villegas, Alejandra

2016 "Sistema posesivo de las partes del cuerpo en mazateco", ponencia presentada en el XI Coloquio de Lingüística en la Escuela Nacional de Antropología e Historia, del 26-29 de abril, Ciudad de México.

Ortiz Villegas, Alejandra, Armando Mora-Bustos, Francisco Arellanes Arellanes y H. Antonio García Zúñiga

2014 "Codificación de relaciones semánticas no eventivas en cuatro lenguas otomangues", ponencia presentada en el Coloquio sobre lenguas otomangues y vecinas 6, del 24-27 de abril de 20I4, Oaxaca, Oaxaca.

Payne, Thomas

2006 Exploring Language Structure. A Student's Guide, Cambridge: Cambridge University Press.

Picallo, Carme y Gemma Rigau

I999 “El posesivo y las relaciones posesivas”, en: Violeta Demonte e Ignacio Bosque (coords.), Gramática descriptiva de la lengua española, vol. I, Madrid: Espasa-Calpe, pp. 973-IO2I.

Pozzi-Escot, Inés

I973 Apuntes sobre el castellano de Ayacucho, Lima: Universidad Nacional de San Marcos.

Risco, Roxana

2013 "Nuevas perspectivas teóricas y empíricas: El doble posesivo de tercera persona", Escritura y pensamiento, XVI(32), pP. 4I-7I. 
Rodríguez Garrido, José Antonio

1982 "Sobre el uso del posesivo redundante en el español de Perú", Lexis, vi(I), Pp. II7-I23.

Seiler, Hansjakob

198I Possession as an Operational Dimension of Language, 42, Köln: Institut für Sprachwiss, Universität Köln.

Shopen, Timothy

1985 Language Typology and Syntactic Description, vols. I, 2, 3, Cambridge: Cambridge University Press.

Taylor, John

1999 "Possession" en: Keith Brown y Jim Miller (eds.), Concise Encyclopedia of Grammatical Categories, Oxford: Elsevier Science, pp. 300-303.

Van Valin, Robert y Randy LaPolla

1997 Syntax: structure, meaning and function. Cambridge: Cambridge University Press.

Armando Mora Bustos

Maestro en Lingüística Española por el Instituto Caro y Cuervo (Colombia) y doctor en Lingüística por el Centro de Estudios Lingüísticos y Literarios de El Colegio de México. Se desempeña como profesor investigador de tiempo completo en la Universidad Autónoma Metropolitana, unidad Iztapalapa. Su línea de investigación es la "gramática descriptiva: interface entre la morfosintaxis, la semántica y la pragmática.

Alejandra Itzel Ortiz Villegas

Licenciada en Comunicación Social por la Universidad Autónoma Metropolitana y en Lengua y Literatura Hispánica por la Universidad Nacional Autónoma de México. También es maestra en Filología por el Instituto de Lengua Española del Consejo Superior de Investigaciones Científicas de España. Actualmente es doctorante en Lingüística por la Universidad Nacional Autónoma de México.

Hamlet Antonio García Zuñiga

Lingüista. Se desempeña como Investigador titular en el Instituto Nacional de Antropología e Historia, en la sede de Yucatán. Sus líneas de investigación son la Sociolingüística y las Tipologías lingüísticas. 
Citar como: Armando Mora-Bustos, Alejandra Itzel Ortiz Villegas y $\mathrm{H}$. Antonio García Zúñiga (2019), "Un determinante con contenido: el caso de su/sus ", Iztapalapa. Revista de Ciencias Sociales y Humanidades, núm. 86, año 40, enero-junio de 20I9, ISSN 2007-9I76; pp. II-40. Disponible en $<$ http://revistaiztapalapa.izt.uam.mx/index.php/izt/issue/archive $>$. 